\title{
A Screw Theory of Static Beams
}

\author{
J.M. Selig and X. Ding* \\ School of Computing, Info. Sys. \& Maths. \\ South Bank University \\ London SE1 0AA, U.K.
}

\begin{abstract}
In this article we derive the deflection equation of a simple beam using screw theory. The effects of tension, torsion and bending of the beam can be unified into a single equation.

We begin by looking at the compliance matrix for small elements of the beam. This is loosely based on work by von Mises in the 1920s. We reproduce von Mises results for the compliance matrix of the entire beam by integrating along the beam. This allows us to make some general comments concerning the use of beams as compliant design element.

Then we derive the equation for the deflection of the beam considering the deflection as a rigid body motion.
\end{abstract}

\section{Introduction}

The idea behind this work is to recast the usual theory of slender beams into a formalism consistent with the theory of the group of rigid-body motions $S E(3)$. We can do this because we make the assumption that the cross-section of the beam doesn't change shape when bent. This is a usual assumption and, like all the assumptions we make, is reasonable if the bending is small and the material is stiff.

Ultimately we want to be able to model the links of a robot as simple beams. Most approaches to the modelling of compliant manipulators only study bending in 2-dimensions. Experience suggests that

\footnotetext{
*permanent address: Robotics Research Institute, Beijing
} University of Aero.\& Astro., P. R. China a full three dimensional model will be facilitated by the use of 'Screw theory', it will allows us to take account of arbitrary torques and reaction wrenches at the ends of the link. We use the term screw theory loosely to mean the theory of the group of rigid-body motions and its Lie algebra.

Von Mises [4] derived the theory of beams using motors or what we would call screws. However, this theory was essentially an infinitesimal theory modelling deformations of the beam as screws. We desire a theory that can cope with larger deformations and eventually with the dynamics of beams. So we will use the von Mises' theory for small elements along the bar and then put the elements together to form bars of finite length. In this article we only look at the static case, the deformation of the bar produced by a static load.

We begin by describing the notation and assumptions we will be making.

\section{Notation}

The un-bent bar will be assumed to lie along the $x$ axis, we assume that it is slender, that is its width will be small compared to any bending radius we impose on the beam. As mentioned above, we assume that the stress on the beam is small so that cross-sections of the beam will be undistorted. This is necessary so that we can assume that small elements undergo rigid transformations.

The von Mises' theory for the bar is referred to a coordinate system in the middle of the bar. For our purposes we need to refer the equations for each 
element to a common coordinate system. We will take this common coordinate system to be at the foot of the beam.

In general we need to know how things transform under rigid motions and rigid changes of coordinates. Let $\mathbf{s}^{T}=\left(\boldsymbol{\omega}^{T}, \mathbf{v}^{T}\right)$ be a screw, that is an element of the Lie algebra of $S E(3)$. Now an active transformation will move the screw according to the relation,

$$
\mathbf{s}^{\prime}=H \mathbf{s}=\left(\begin{array}{cc}
R & 0 \\
T R & R
\end{array}\right)\left(\begin{array}{l}
\boldsymbol{\omega} \\
\mathbf{v}
\end{array}\right)
$$

where $R$ is the rotation matrix of the transformation and $T$ is the anti-symmetric matrix representing the translation, $(T \mathbf{x}=\mathbf{t} \times \mathbf{x})$. The transformation matrix $H$ here is an element of the adjoint representation of the group $S E(3)$.

The corresponding change of coordinates by the same rotation and translation is given by the inverse of the active transformation matrix $H$,

$$
\overline{\mathbf{s}}=H^{-1} \mathbf{s}=\left(\begin{array}{cc}
R^{T} & 0 \\
-R^{T} T & R^{T}
\end{array}\right)\left(\begin{array}{l}
\boldsymbol{\omega} \\
\mathbf{v}
\end{array}\right)
$$

A coordinate transformation like this is sometimes refered to as a passive transformation.

A wrench is an element of the dual to the Lie algebra, a general wrench can be written as $\mathcal{W}^{T}=$ $\left(\boldsymbol{\tau}^{T}, \mathbf{F}^{T}\right)$. To keep the scalar $\mathcal{W}^{T} \mathbf{s}$ coordinate invariant, wrenches must transform under the inverse transpose representation of $H$ for an active transformation and the transpose of $H$ for a coordinate change,

$$
\begin{aligned}
\mathcal{W}^{\prime}=H^{-T} \mathcal{W}= & \left(\begin{array}{cc}
R & T R \\
0 & R
\end{array}\right)\left(\begin{array}{c}
\boldsymbol{\tau} \\
\mathbf{F}
\end{array}\right), \\
& \overline{\mathcal{W}}=H^{T} \mathcal{W}=\left(\begin{array}{cc}
R^{T} & -R^{T} T \\
0 & R^{T}
\end{array}\right)\left(\begin{array}{c}
\boldsymbol{\tau} \\
\mathbf{F}
\end{array}\right)
\end{aligned}
$$

Finally here we look at the transformation of a compliance matrix under a passive change of coordinates. The compliance matrix transforms a wrench into a screw so under a rigid coordinate change a compliance matrix $C$ will transform according to the relation,

$$
\bar{C}=H^{-1} C H^{-T}
$$

For example if, the new coordinate system is a translation by $\mu$ units along the $x$-axis of the old one, the corresponding change of coordinate will be given by the above with,

$$
H^{-1}=\left(\begin{array}{cccccc}
1 & 0 & 0 & 0 & 0 & 0 \\
0 & 1 & 0 & 0 & 0 & 0 \\
0 & 0 & 1 & 0 & 0 & 0 \\
0 & 0 & 0 & 1 & 0 & 0 \\
0 & 0 & \mu & 0 & 1 & 0 \\
0 & -\mu & 0 & 0 & 0 & 1
\end{array}\right)
$$

and so forth.

\section{3 von Mises' Elements}

In this section we will review von Mises' theory which we will use for small elements of the bar.

The element is in equilibrium so when the only load is a wrench at the tip of the beam, we assume it is subject to a pair of wrenches $\mathcal{W}$ and $-\mathcal{W}$. Here $\mathcal{W}$ is the wrench imposed by the next element and ultimately by the tip wrench. While $-\mathcal{W}$ is imposed by the previous element from Newton's 3-rd law.

The effect of this stress on the bar is to cause small deflections of the ends of the elements. These small deflections will be measured by screws $\mathbf{s}_{1}$ and $\mathbf{s}_{2}$. The wrench $\mathcal{W}$, which produces this small deformation will be given by the relation,

$$
C \mathcal{W}=\left(\mathbf{s}_{2}-\mathbf{s}_{1}\right)
$$

Where $C$ is the compliance matrix of the element. To find the elements of the compliance matrix for the element we look at particular loads.

We begin with the simple case of tension or compression along the beam. So assume a force $F_{x}$ along the $x$-axis. If the length of the element is $\Delta \mu$ and the magnitude of the deflection is $\Delta x$, then from elementary mechanics we have,

$$
F_{x} \frac{1}{E A}=\frac{\Delta x}{\Delta \mu}
$$

where $E$ is Young's modulus and $A$ is the crosssectional area of the beam.

The next case is a simple torsion about the length of the beam. If the torque about the $x$-axis is $\tau_{x}$ and 


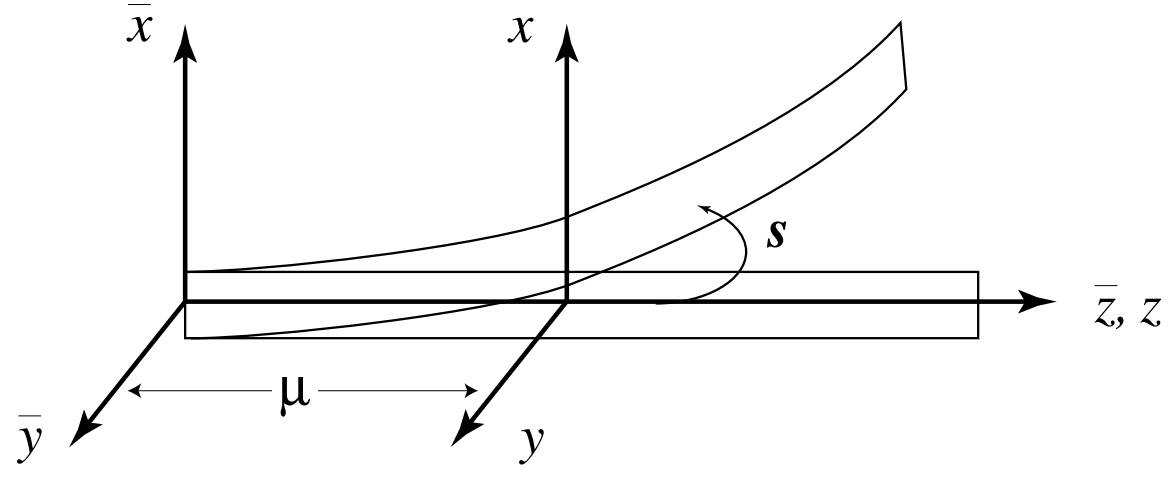

Figure 1: A Small Element in the Beam

the corresponding angular deflection of the beam is $\Delta \phi_{x}$ then,

$$
\tau_{x} \frac{1}{G J}=\frac{\Delta \phi_{x}}{\Delta \mu}
$$

where $G$ is the shear modulus and $J$ is a quantity relating to the cross-section of the beam.

Our third case concerns pure bending, we apply a bending moment $\tau_{y}$ about the $y$-axis, the result is an angular deflection $\phi_{y}$. We get,

$$
\tau_{y} \frac{1}{E I_{z}}=\frac{\Delta \phi_{y}}{\Delta \mu}
$$

where $I_{z}$ is the second moment of area for the beam, see fig.1. Clearly a similar result applies to bending about the $z$-axis.

The final case to consider is the result of forces in the $y$ and $z$ directions. these forces pass through the centre of the bar and it is easy to see that they produce negligible deflection at the ends of the bar.

Putting all this together we get the equation,

$$
c\left(\begin{array}{c}
\tau_{x} \\
\tau_{y} \\
\tau_{z} \\
F_{x} \\
F_{y} \\
F_{z}
\end{array}\right)=\frac{1}{\Delta \mu}\left(\begin{array}{c}
\Delta \phi_{x} \\
\Delta \phi_{y} \\
\Delta \phi_{z} \\
\Delta x \\
\Delta y \\
\Delta z
\end{array}\right)
$$

Where the matrix $c$ is,

$$
c=\left(\begin{array}{cccccc}
\frac{1}{G J} & 0 & 0 & 0 & 0 & 0 \\
0 & \frac{1}{E I_{z}} & 0 & 0 & 0 & 0 \\
0 & 0 & \frac{1}{E I_{y}} & 0 & 0 & 0 \\
0 & 0 & 0 & \frac{1}{E A} & 0 & 0 \\
0 & 0 & 0 & 0 & 0 & 0 \\
0 & 0 & 0 & 0 & 0 & 0
\end{array}\right)
$$

This is a sort of compliance density for the element. That is the compliance matrix for the element is $C=$ $c \Delta \mu$.

\section{Compliance Matrix of a Beam}

An immediate application of the above is to compute the compliance matrix for a long slender beam. It is well known that for serial devices the total compliance matrix is the sum of the compliance matrices for individual elements. Hence we can find the total compliance matrix for a beam by integrating along the bar. We must ensure that all the compliance matrices for the elements are expressed in a common coordinate frame. Here we choose the coordinate frame in the middle of the bar.

Assume the bar has length $l$, an element a distance $\mu$ from the end of the bar will have compliance matrix, $C(\mu)=c(\mu) \Delta \mu=H^{-1} C H^{-T}$, where $H$ is 
the active transformation to the standard coordinate frame. When the standard frame is in the middle of the bar, this transformation is a pure translation, $(l / 2-\mu)$ in the $x$-direction. Substituting this in the relation for the coordinate transformation gives,

$c(\mu)=\left(\begin{array}{cccccc}\frac{1}{G J} & 0 & 0 & 0 & 0 & 0 \\ 0 & \frac{1}{E I_{z}} & 0 & 0 & 0 & \frac{2 \mu-l}{2 E I_{z}} \\ 0 & 0 & \frac{1}{E I_{y}} & 0 & \frac{l-2 \mu}{2 E I_{y}} & 0 \\ 0 & 0 & 0 & \frac{1}{E A} & 0 & 0 \\ 0 & 0 & \frac{2 \mu-l}{2 E I_{y}} & 0 & \frac{(2 \mu-l)^{2}}{4 E I_{y}} & 0 \\ 0 & \frac{l-2 \mu}{2 E I_{z}} & 0 & 0 & 0 & \frac{(2 \mu-l)^{2}}{4 E I_{z}}\end{array}\right)$

Now we can integrate with respect to $\mu$ from 0 to $l$ we get the result,

$$
C_{t o t}=\left(\begin{array}{cccccc}
\frac{l}{G J} & 0 & 0 & 0 & 0 & 0 \\
0 & \frac{l}{E I_{z}} & 0 & 0 & 0 & 0 \\
0 & 0 & \frac{l}{E I_{y}} & 0 & 0 & 0 \\
0 & 0 & 0 & \frac{l}{E A} & 0 & 0 \\
0 & 0 & 0 & 0 & \frac{l^{3}}{12 E I_{y}} & 0 \\
0 & 0 & 0 & 0 & 0 & \frac{l^{3}}{12 E I_{z}}
\end{array}\right)
$$

This result agrees with the result given in von Mises [4], which was derived by slightly different methods. It was also given in [1]

This prompts an intriguing design question: Given a diagonal compliance (or stiffness) matrix, can one always find a beam with the same matrix? There appear to be seven parameters specifying the compliance matrix of the beam, $l$ the length of the beam, $E$ and $G$ the Young's and shear modulus and the crosssectional area quantities $A, I_{z}, I_{y}$ and $J$. However, there is an obvious consistency condition. Suppose that the target compliance matrix has diagonal entries $x_{1}, x_{2}, x_{3}, u_{1}, u_{2}, u_{3}$, we can find the length of the bar in two ways, $l^{2}=12\left(u_{3} / x_{2}\right)=12\left(u_{2} / x_{3}\right)$. So for the compliance matrix to be representable by a beam it must satisfy $x_{2} u_{2}-x_{3} u_{3}=0$ and thus not all diagonal compliance matrices come from single beams.

To make further progress with the above question, notice that all beams of the type we have been looking at can be represented by beams with rectangular cross-sections. Suppose the rectangle has sides $a$ and $b$ then the area quantities are, $A=a b, I_{z}=a^{3} b / 12$, $I_{y}=a b^{3} / 12$ and $J=a b\left(a^{2}+b^{2}\right) / 12$. So that, $a=\sqrt{12 u_{1} / x_{2}}$ and $b=\sqrt{12 u_{1} / x_{3}}$. The value of Young's modulus is given by $E=u_{1}^{2} \sqrt{12 / x_{2} u_{2}}=$ $u_{1}^{2} \sqrt{12 / x_{3} u_{3}}$. Finally, since $E / G=2(1-\sigma)$ we can find Poisson's ratio $\sigma$ from the quotient $x_{1} / u_{1}$. After simplification we get that, $\sigma=\left(2 x_{2} x_{3}-x_{1} x_{2}-\right.$ $\left.x_{1} x_{3}\right) / 2 x_{2} x_{3}$.

This gives a physical constraint, since the Poisson's ratio must be positive and less than $1 / 2$. Of course it is unlikely that there will be a material with the particular properties found in this way.

A more practical approach might be to use more than one beam to achieve a given stiffness matrix. Can any stiffness matrix be designed by using several of these beams in parallel? The answer to this is clearly no. Consider the quantity $b_{1}=\operatorname{Tr}\left(Q_{0} K\right)$, where $K$ is a stiffness matrix and $Q_{0}=\left(\begin{array}{cc}0 & I_{3} \\ I_{3} & 0\end{array}\right)$ in partitioned form. It is possible to show that this quantity is invariant with respect to rigid coordinate changes, see [6]. Clearly, for our simple beams we have that $b_{1}=0$, moreover since the trace operator is additive and sum of stiffness matrices resulting from putting beams in parallel will also have $b_{1}=0$. Notice that the straightforward eigenvalues of the stiffness matrix don't have much significance because they are not invariant with respect to coordinate changes and hence will have different valuse in different coordinate frames.

Huang and Schimmels [3] showed that any stiffness matrix could be achieved using simple springs connected in parallel provided $b_{1}=0$ for the stiffness matrix. Hence the of any system of beams can be simulated with simple springs. But we cannot achieve any more with beams than we could with simple springs. It is not clear at present whether or not there is an equivalence between beams and springs as design elements. That is, can design systems of beams with exactly the same stiffness matrix as any system of simple springs? However, in many cases it may be advantageous to use beams rather than springs since probably fewer of them would required. 


\section{Deflection Equation}

The deflection of the bar will be represented by an element from the group of rigid-body transformations. As we move along the bar we imagine a small element of the bar is subject to a rigid transformation. Hence the shape of the bar will be given by a function $g(\mu)$ where $\mu$ is a parameter which measures the distance along the bar in its un-stressed state and $g \in S E(3)$. This can be thought of as a path in the group space, see fig. 2 .

Since the rigid-body motions form a Lie group, we can use the exponential map to write the deflection as a function on the Lie algebra,

$$
g(\mu)=e^{\mathbf{s}(\mu)}
$$

the Lie algebra element $\mathbf{s}$ here will be referred to as the deflection screw. Clearly if we find the deflection screw for the beam as a function of the parameter $\mu$ then it is a simple matter to compute the deflection of the bar described by the path in the group space, $g(\mu)$. The ability to use the logarithm function to 'pull-back' the path in the group to a path in the Lie algebra relys on the assumption that the deflection is 'small'.

Now from section 3 we have that for an element in the bar $C \mathcal{W}=\left(\mathbf{s}_{2}-\mathbf{s}_{1}\right)$. The screws here represent small changes in the deflection of the ends of the beam measured in a coordinate frame in the middle of the element. If the deflection is small then the group element representing the deflection can be written as $g(\mu) \approx I+\mathbf{s}(\mu)$, so the deflection at the ends of the bar can be written as,

$$
\mathbf{s}_{1}=\mathbf{s}(\mu-\Delta \mu / 2), \quad \mathbf{s}_{2}=\mathbf{s}(\mu+\Delta \mu / 2)
$$

Hence the quantity, $\left(\mathbf{s}_{2}-\mathbf{s}_{1}\right) / \Delta \mu$ becomes $d \mathbf{s} / d \mu$ as the elements get shorter. Notice that the derivative of the deflection screw, $d \mathbf{s} / d \mu$ is also a screw that is an element of the Lie algebra. If we divide the original equation $C \mathcal{W}=\left(\mathbf{s}_{2}-\mathbf{s}_{1}\right)$, by $\Delta \mu$ and take the limit as $\Delta \mu \rightarrow 0$ we get the differential equation, $d \mathbf{s} / d \mu=c \mathcal{W}$.

This equation is expressed in a coordinate system in the middle of the element, to express it in a coordinate system at the foot of the beam only the compliance matrix for the element needs to be transferred to this frame. The active transformation from this local frame to the frame at the foot of the beam is given by, $e^{-\mu \mathbf{t}} e^{-\mathbf{s}(\mu)}$, where $\mathbf{t}$ is a unit translation along the bar in the $x$-direction.

Using the results given in sections 2 and 3 we find the deflection equation for the beam is,

$$
\frac{d \mathbf{s}(\mu)}{d \mu}=e^{\mathbf{s}(\mu)} e^{\mu \mathbf{t}} c e^{\mu \mathbf{t}^{T}} e^{\mathbf{s}^{T}(\mu)} \mathcal{W}
$$

It is not too difficult to see how this can be extended to more general systems of wrenches acting on the beam and also to systems of distributed wrenches such as the weight of the beam.

\section{An example - The Planar Beam}

To validate our work we look at a standard example, the deflection of a cantilever beam with a concentrated load at its tip. Because the case is planar we use a $3 \times 3$ formalism, we can use the $3 \mathrm{rd}$, 4 th and 5 th components of the representation of the spatial motions group used above.

A point $(x, y)$ in the plane is represented by the 3 -vector, $(x, y, 1)^{T}$ but a Lie algebra element is represented by a 3 -vector $\mathbf{s}=\left(\theta, t_{x}, t_{y}\right)^{T}$. For the concentrated load $F$ the wrench is $\mathcal{W}=(l F, 0, F)^{T}$. The exponential of a screw $\mathbf{s}$, can be written as a $3 \times 3$ transformation matrix,

$$
e^{\mathbf{s}}=\left(\begin{array}{ccc}
1 & 0 & 0 \\
c_{y} & \cos \theta & -\sin \theta \\
-c_{x} & \sin \theta & \cos \theta
\end{array}\right)
$$

where, $c_{x}=\frac{t_{x}}{\theta} \sin \theta-\frac{t_{y}}{\theta}(1-\cos \theta)$, and $c_{y}=\frac{t_{x}}{\theta}(1-$ $\cos \theta)+\frac{t_{y}}{\theta} \sin \theta$.

The compliance density of an element is given by the matrix,

$$
\left.c_{(} \mu\right)=e^{\mu \mathbf{t}} c e^{\mu \mathbf{t}^{T}}=\left(\begin{array}{ccc}
\frac{1}{E I} & 0 & \frac{-\mu}{E I} \\
0 & \frac{1}{E A} & 0 \\
\frac{-\mu}{E I} & 0 & \frac{\mu^{2}}{E I}
\end{array}\right)
$$

where, $I=I_{y}$ is the second moment of area, for planar problems only one is relevant. 
Substituting this into the deflection equation gives 3 non-linear coupled differential equations,

$$
\begin{aligned}
\theta^{\prime}= & \frac{F}{E I}\left(l-\mu \cos \theta-c_{x}\right) \\
t_{x}^{\prime}= & \frac{F}{E I}\left(l c_{y}-c_{x} c_{y}-\mu c_{y} \cos \theta-\mu^{2} \cos \theta \sin \theta+\right. \\
& \left.\mu l \sin \theta-\mu c_{x} \sin \theta\right)+\frac{F}{E A} \cos \theta \sin \theta \\
t_{y}^{\prime}= & \frac{F}{E I}\left(\mu^{2} \cos ^{2} \theta-\mu l \cos \theta+2 \mu c_{x} \cos \theta-\right. \\
& \left.l c_{x}+c_{x}^{2}\right)+\frac{F}{E A} \sin ^{2} \theta
\end{aligned}
$$

In line with the classical theory we introduce some additional assumptions here. We suppose that the rotation angle $\theta$ is small, so $\cos \theta \approx 1, \sin \theta \approx \theta$ and $\sin ^{2} \theta \approx 0$. Also assume $c_{x} \approx 0$ this means that the motion of each element is only in the $y$-direction. With these assumptions the first and last equations become,

$$
\theta^{\prime}=\frac{F}{E I}(l-\mu) \quad t_{y}^{\prime}=\frac{F}{E I}\left(\mu^{2}-\mu l\right)
$$

Using the initial conditions $t_{y}(0)=0$ and $\theta(0)=0$ the solutions to these equations are simply,

$$
\theta=\frac{F}{E I}\left(l \mu-\frac{\mu^{2}}{2}\right), \quad t_{y}=\frac{F}{E I}\left(\frac{\mu^{3}}{3}-\frac{\mu^{2} l}{2}\right)
$$

To compare this with the classical case we need to know the curve described by the neutral axis of the beam. With no load this is simply the $x$-axis, but when the beam is loaded we need to use the displacement screw to find the curve,

$$
\left(\begin{array}{l}
x \\
y \\
1
\end{array}\right)=\left(\begin{array}{ccc}
\cos \theta & -\sin \theta & c_{x} \\
\sin \theta & \cos \theta & c_{y} \\
0 & 0 & 1
\end{array}\right)\left(\begin{array}{l}
\mu \\
0 \\
1
\end{array}\right)
$$

With the same approximations as above we get that $y=t_{y}+\mu \theta$ since $c_{y} \approx t_{y}$. Hence,

$$
y=\frac{F}{E I}\left(\frac{\mu^{2} l}{2}-\frac{\mu^{3}}{6}\right)
$$

in agreement with the classical texts, see [2] for example.

\section{Conclusions}

In the above we have derived an equation for the deflection of a slender beam. The equation includes the effects due to any applied wrench. Comparisons with existing approximate solutions are not completly straightforward but are possible. Analytic solutions to the equation appear to be difficult to find however, numerical solutions seem to be quite tractable.

When an equation such as the deflection equation is solved numerically it is always difficult to know how accurate the solution is. This is because there is no Riemannian metric defined in the group or rather there are many metrics to choose from and no particular reason to choose any one of them. For the elastic beam there is an obvious natural choice of metric which we can use to compare our solutions, it is given by the elastic energy of the beam.

In future work we hope to explore this and the question of the dynamics of such beams. It is the dynamics of these beams that we hope will provide a simple model for the flexible links of robots.

There are also indirect applications to robotics. The deflection equation represents an equation for a path in the Lie algebra or, by exponentiation, the group itself. Lately there has been much interest in curves in the group for motion interpolation. Originally spline curves were derived from elastic beams, hence it is not unreasonable to propose that a natural generalisation of spline curves to the group is given by solutions to the deflection equation with suitable boundary conditions.

\section{References}

[1] E.D. Fasse and P.C. Breedveld. Modeling of Elastically Coupled Bodies: Part I - General Theory and Geometric Potential Function Method. ASME J. of Dynamical Systems, Measurement, and Control, 120:496-500, 1998.

[2] J.P. Den Hartog. Strength of Materials Dover Publishing, New York, 1961. 
[3] S. Huang and J.M. Schimmels. The bounds and realization of spatial stiffnesses achieved with simple springs connected in parallel. IEEE Trans. on Robotics and Automation, 14(3):466-475, 1998.

[4] R. von Mises. Motorrechnung, ein neues Hilfsmittel in der Mechanik, Zeitschrift für Angewandte Mathematik und Mechanik Band 4, Heft 2, S.155181, 1924. English Translation by E.J. Baker and K. Wohlhart. Motor Calculus, a New Theoretical Device for Mechanics. Institute for Mechanics, University of Technology Graz, Austria, 1996.

[5] J.M. Selig. Geometrical Methods in Robotics. Springer Verlag, New York, 1996.

[6] J.M. Selig and X. Ding. Structure of the Spatial Stiffness Matrix. submitted to IJ Robotics and Automation, 2000. 